\title{
P-glycoprotein inhibition increases the transport of dauricine across the blood-brain barrier
}

\author{
PEI-LIANG DONG ${ }^{1 *}$, HUA HAN $^{1 *}$, TIAN-YU ZHANG ${ }^{1}$, BINGYOU YANG $^{1}$, \\ QIU-HONG WANG ${ }^{1}$ and GAO-WA EERDUN ${ }^{2}$ \\ ${ }^{1}$ Key Laboratory of Chinese Materia Medica of Ministry of Education, Heilongjiang University of Chinese Medicine, \\ Harbin, Heilongjiang 150040; ${ }^{2}$ Department of Pharmacology, The First Hospital of Hebei Medical University, \\ Shijiazhuang, Hebei 050031, P.R. China
}

Received July 17, 2013; Accepted December 12, 2013

DOI: $10.3892 / \mathrm{mmr} .2013 .1880$

\begin{abstract}
Dauricine is the major bioactive component isolated from the roots of Menispermum dauricum D.C. The aim of the present study was to investigate the role of P-glycoprotein in the transport of dauricine across the blood-brain barrier by pre-treatment with the P-glycoprotein inhibitor verapamil. Sprague Dawley rats were divided into a verapamil group (pretreated with verapamil at a dose of $20 \mathrm{mg} / \mathrm{kg}$ ) and a control group (pretreated with the same volume of normal saline). After $90 \mathrm{~min}$, the animals were injected intravenously with dauricine $(10 \mathrm{mg} / \mathrm{kg})$. At 15, 30 and $60 \mathrm{~min}$ after dauricine administration, the levels of dauricine in the blood and brain were detected by high-performance liquid chromatography. Compared with the control group, the dauricine concentration in the brains of the rats in the verapamil group was significantly increased. Furthermore, the brain-plasma ratio of dauricine in the rats pretreated with verapamil was significantly higher than that of the animals in the control group. However, there was no difference identified between dauricine levels in the plasma of the verapamil and the control groups. The results indicated that dauricine is able to pass the blood-brain barrier, and that P-glycoprotein has an important role in the transportation of dauricine across the blood-brain barrier.
\end{abstract}

\section{Introduction}

The blood-brain barrier (BBB) is a diffusion barrier essential for the normal function of the central nervous system (CNS). The BBB has a key role in maintaining homeostasis within the CNS, preserving the composition of the internal milieu,

Correspondence to: Dr Gao-Wa Eerdun, Department of Pharmacology, The First Hospital of Hebei Medical University, Shijiazhuang, Hebei 050031, P.R. China

E-mail: gaowa2001@163.com

*Contributed equally

Key words: dauricine, P-glycoprotein, blood-brain barrier despite variations in the periphery, and protecting the brain against toxins, bacteria and viruses. Moreover, it regulates the uptake of endogenous molecules and xenobiotics into the brain (1). Drug delivery to the CNS is one of the major hurdles in the development of novel therapeutics for neuropsychiatric disorders. In particular, there is a requirement for transport across the BBB, which separates the circulating blood from the $\mathrm{CNS}$, for centrally acting drugs to achieve therapeutic concentrations at their site of action. Of note, $>98 \%$ of potential CNS drugs are unable to cross the $\mathrm{BBB}$ to reach target sites within the brain (2). The BBB is the bottleneck in brain drug development and is the single most important factor limiting future development of neurotherapeutics (3).

P-glycoprotein is a plasma membrane glycoprotein that is able to exclude a wide range of chemotherapeutic drugs and other hydrophobic compounds from cells (4). It is also expressed at high levels in non-cancerous tissues, such as the endothelial cells of BBB capillaries in humans, as well as animals. P-glycoprotein may be involved in the exclusion of various drugs from the capillary endothelial cells, blocking their entry into the brain (5). Verapamil is the most extensively characterized P-gp inhibitor and multidrug resistance-associated protein (MDR) reversal agent (6).

Dauricine (Fig. 1) is a bisbenzylisoquinoline alkaloid isolated from the root of Menispermum dauricum D.C. Dauricine has been suggested for the treatment of various diseases, including cardiac ischemia, angina and inflammation $(7,8)$. Previous studies have demonstrated that dauricine inhibits angiogenesis in tumors (9) and promotes apoptosis in tumor cells (10). A study by Li and Gong (11) suggested that dauricine exhibits neuroprotective action in rats subjected to transient focal cerebral ischemia by attenuating apoptosis in the ischemic penumbra (12). However, it has yet to be elucidated whether dauricine is capable of crossing the BBB. The main purpose of the present study was thus to investigate whether dauricine is able to cross the BBB by assessing dauricine levels in the plasma and brain tissue of rats.

\section{Materials and methods}

Drugs and chemicals. Verapamil, a P-glycoprotein inhibitor, was obtained from Shanghai Hefeng Pharmaceutical Co., 
Ltd. (Shanghai, China). Tetrandrine was obtained from the National Institute for the Control of Pharmaceutical and Biological Products. AB-8 cross-linked polystyrene and silica gel $\left(\mathrm{SiO}_{2}\right)$ were obtained from Nankai University (Professor Li, Ministry of Education, Heilongjiang Key Laboratory of TCM Pharmacodynamic Material Bases, Tianjin, China). Acetonitrile and methanol [high-performance liquid chromatography (HPLC) grade] were obtained from Merck KGaA (Darmstadt, Germany). Triethylamine, phosphoric acid, and dichloromethane (analytical grade) were purchased from Tianjin Bodi Chemical Co., Ltd. (Tianjin, China).

Dauricine preparation. The dried roots ( $5 \mathrm{~kg}$ ) of Menispermum dauricum D.C were extracted with $70 \% \mathrm{EtOH}$ under reflux (2x 50 liters) for $2.5 \mathrm{~h}$. The combined solution was filtered and concentrated under vacuum to give a syrup, followed by resuspension in distilled water. The suspension was passed through AB-8 cross-linked polystyrene, and sequentially eluted with distilled water and 50\% EtOH. The extraction eluted with $50 \% \mathrm{EtOH}$ was concentrated under vacuum to yield a syrup $(56.0 \mathrm{~g})$. This crude residue was subjected to column chromatography (CC) on $\mathrm{SiO}_{2}$ with a mobile phase of $\mathrm{CHCl}_{3} / \mathrm{MeOH}(10: 1 \rightarrow 5: 1)$, which gained five fractions (Fr. 1-5). Fr. 3-4 (10.4 g) were subjected to $\mathrm{CC}$ on $\mathrm{SiO}_{2}$ $\left(\mathrm{CHCl}_{3} / \mathrm{MeOH}, 8 \rightarrow 1\right.$; then octadecylsilyl (ODS), $\mathrm{MeOH} / \mathrm{H}_{2} \mathrm{O}$, 1:1) to yield three substances. Each substance was purified by preparative HPLC on a Hypersil-ODS II column (10 $\mu \mathrm{m}$, 20x300 mm, flow rate $8 \mathrm{ml} / \mathrm{min}$; DIMKA Co., Beijing, China), with $\mathrm{MeOH} / \mathrm{H}_{2} \mathrm{O}(70: 30)$ as the eluent, with a retention time of $t_{R}=15.0$ min. Fig. 2 shows the typical HPLC chromatogram of dauricine with a purity $>96 \%$.

Animals and treatment. Forty-eight male Sprague Dawley rats weighing $220 \pm 20 \mathrm{~g}$ were provided by the Experimental Animal Center of Heilongjiang University of Chinese Medicine (Harbin, China). The certificate number was 2008004. All experimental procedures conducted in this study were performed in accordance with the guidelines for the Care and Use of Laboratory Animals of Heilongjiang University of Chinese Medicine. The rats were kept with free access to food and water and a $12 \mathrm{~h}$ light/dark cycle. They were housed in plastic cages and randomly divided into two groups: The control and verapamil groups, containing 24 animals each. The rats in the verapamil group were administered intraperitoneally (i.p.) with verapamil at a dose of $20 \mathrm{mg} /$ $\mathrm{kg}$. The rats in the control group were treated with an equal volume of normal saline. After $90 \mathrm{~min}$, all rats were injected with dauricine $(15 \mathrm{mg} / \mathrm{kg})$ through the tail vein. At 15,30 and $60 \mathrm{~min}$ after dauricine treatment, the animals were anesthetized with chloral hydrate $(300 \mathrm{mg} / \mathrm{kg}$, i.p.) and then $5 \mathrm{ml}$ heparinized blood add $100 \mu \mathrm{l}$ of $1 \%$ heparin (Tianjin Biochem Pharmaceutical Company, Tianjin, China ) into the blood] was collected from the abdominal aorta. The blood samples were centrifuged $\left(3,500 \mathrm{x} \mathrm{g}, 4^{\circ} \mathrm{C}\right)$ for $10 \mathrm{~min}$ and the plasma was separated. A $600-\mu 1$ aliquot of plasma was added to $1,200 \mu 1$ methanol. Subsequent to vortexing, $3 \mathrm{ml}$ dichloromethane was added, and the mixture was vortexed for $3 \mathrm{~min}$ and centrifuged at 3,500 $\mathrm{xg}$ for $10 \mathrm{~min}$. The organic layer was removed and evaporated to dryness at $40^{\circ} \mathrm{C}$ under a stream of nitrogen.

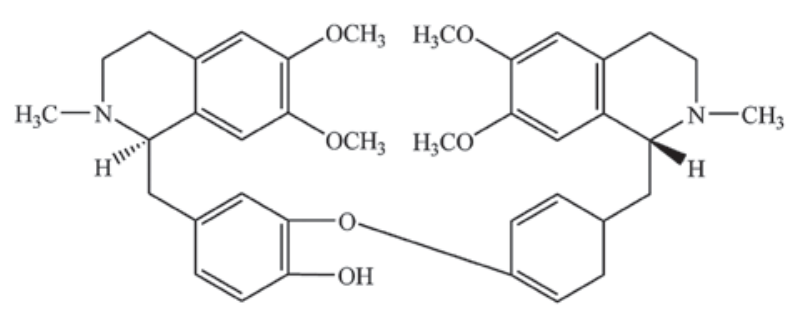

Figure 1 The chemical structure of dauricine

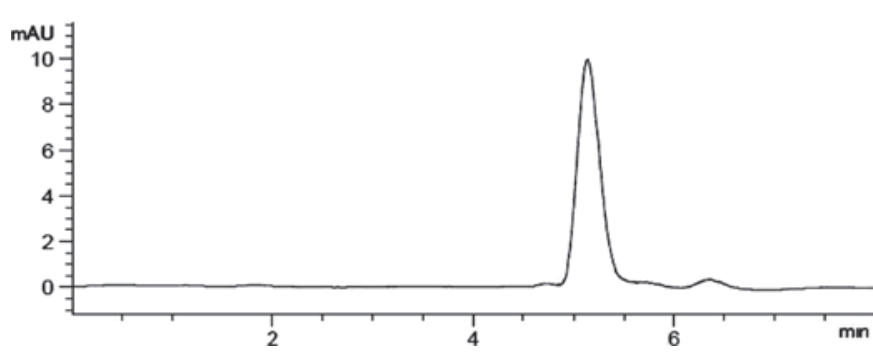

Figure 2. High-performance liquid chromatogram of dauricine.

The residue was dissolved in $600 \mu 1$ mobile phase, and $10 \mu \mathrm{l}$ supernatant liquid was injected into the HPLC system. The rats were perfused with $100 \mathrm{ml}$ ice-cold normal saline each. The brain was instantly removed from the cranium and weighed. The brain was then homogenized in four aliquots of $0.1 \mathrm{~mol} / \mathrm{l}$ ice-cold phosphate buffer ( $\mathrm{pH}$ 7.4). Dichloromethane (3 ml) was added to $600 \mu 1$ homogenate. Following vortexing for $3 \mathrm{~min}$ and centrifuging $\left(3,500 \mathrm{xg}, 4^{\circ} \mathrm{C}\right)$ for $10 \mathrm{~min}$, the supernatants were evaporated to dryness under a stream of nitrogen at $40^{\circ} \mathrm{C}$. The residue was analyzed according to the method outlined in the proceeding section.

Chromatographic conditions. The chromatographic separation was performed using an Agilent 1260 Series HPLC system (Agilent Technologies, Santa Clara,CA,USA).The HPLC system consisted of a quaternary pump, an ultraviolet detector operated at $281 \mathrm{~nm}$ and a Diamonsil C18 column (250x4.6 mm, $5 \mu \mathrm{m})$ protected with a guard column packed with the same material. The column was kept at room temperature. The mobile phase was acetonitrile- $0.4 \%$ phosphate buffer $(20: 80, \mathrm{v} / \mathrm{v})$. The pump was operated at a flow rate of $1 \mathrm{ml} / \mathrm{min}$.

Statistical analysis. Data are expressed as the mean \pm standard deviation. The statistical significances of the data were determined using one-way analysis of variance followed by Least Significant Difference testing. $\mathrm{P}<0.05$ was considered to indicate a statistically significant difference.

\section{Results}

HPLC of dauricine. Fig. 3 shows typical HPLC chromatograms of the brains of untreated rats, brain spiked with dauricine (standard) and tetrandrine (internal standard, No. 110711; National Institutes for Food and Drug Control, Beijing, China), and brain of rats treated with dauricine and spiked with tetrandrine (internal standard). Fig. 4 shows typical HPLC chromatograms of plasma from untreated rats, plasma spiked with dauricine and tetrandrine, and plasma of 


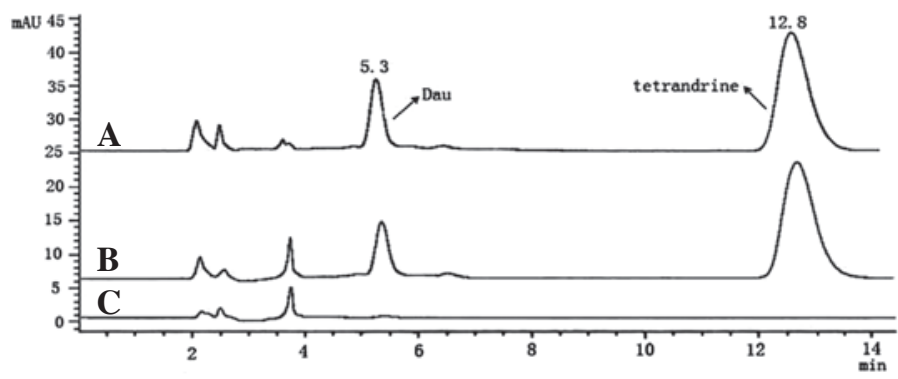

Figure 3. High-performance liquid chromatograms of rat brain. (A) Brain of rat treated with dauricine and spiked with tetrandrine (internal standard). (B) Blank brain sample spiked with dauricine (standard) and tetrandrine (internal standard). (C) Blank brain sample.

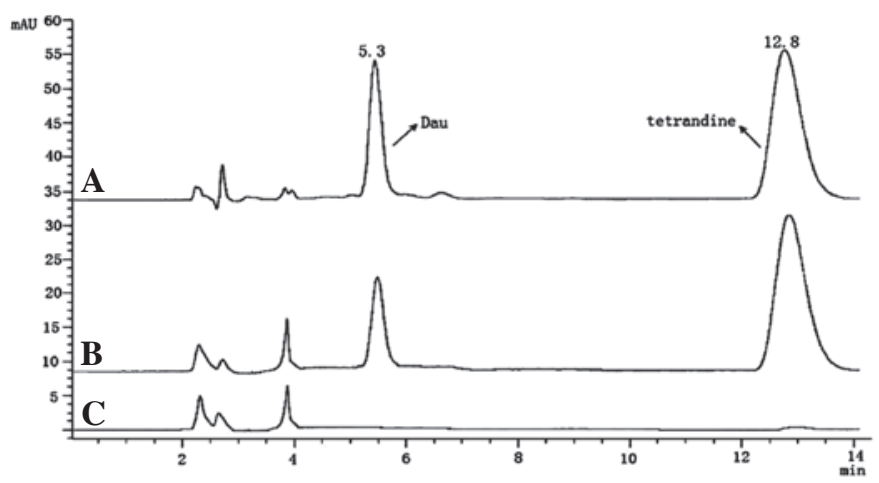

Figure 4. High-performance liquid chromatograms of rat plasma. (A) Plasma of rat treated with dauricine and spiked with tetrandrine (internal standard). (B) Blank plasma sample spiked with dauricine (standard) and tetrandrine (internal standard). (C) Blank plasma sample.

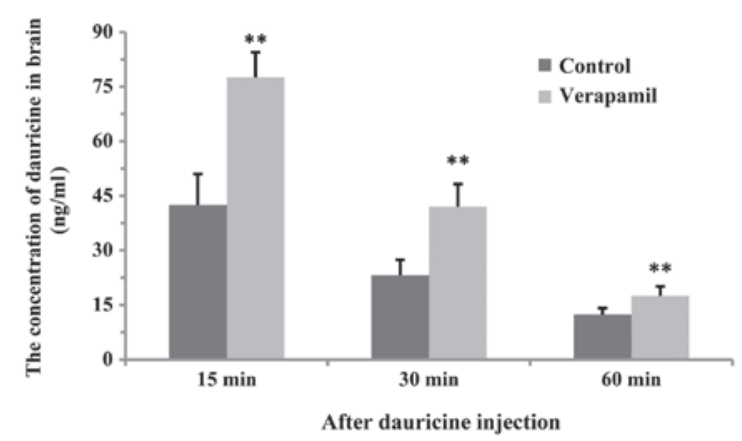

Figure 5. Effect of verapamil on dauricine levels in rat brains. Data are expressed as the mean \pm standard error of the mean $(n=8)$. Statistical significances are determined using one-way analysis of variance followed by Least Significant Difference testing. ${ }^{* *} \mathrm{P}<0.01$ compared with the control group.

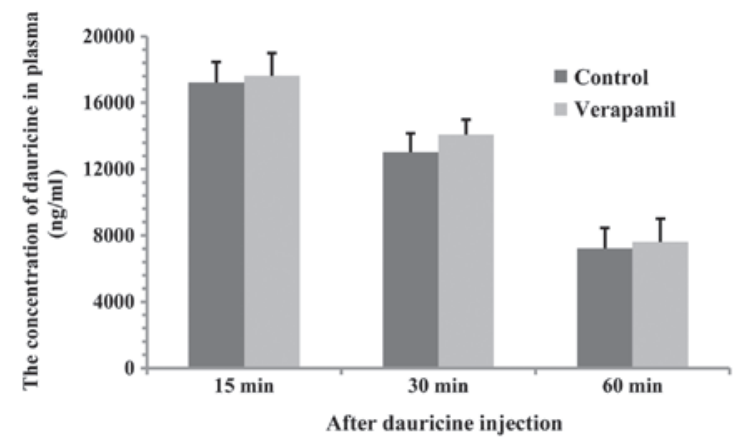

Figure 6. Effect of verapamil on dauricine levels in rat plasma. Data are expressed as the mean \pm standard error of the mean $(n=8)$. Statistical significances were determined using one-way analysis of variance followed by Least Significant Difference testing.

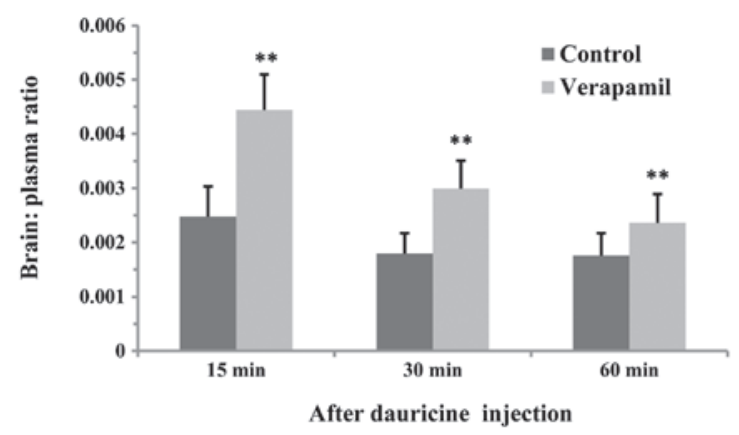

Figure 7. Effect of verapamil on the brain:plasma ratio of dauricine concentration in rats. Data are expressed as the mean \pm standard error of the mean $(n=8)$. Statistical significances were determined using one-way analysis of variance followed by Least Significant Difference testing. ${ }^{* *} \mathrm{P}<0.01$ compared with the control group.

rats treated with dauricine and spiked with tetrandrine. The retention times of dauricine and tetrandrine were 5.3 and $12.8 \mathrm{~min}$, respectively, where tetrandrine was the internal standard.

Effect of verapamil on the dauricine concentration in the brain. At 15,30 and 60 min after dauricine treatment, dauricine levels in the brain of the verapamil group was significantly increased compared with those of the control group ( $\mathrm{P}<0.01$, Fig. 5).

Effect of verapamil on dauricine levels in plasma. Compared with the control, pretreatment with verapamil exerted no significant effect on dauricine levels in plasma (Fig. 6). 
Effect of verapamil on the dauricine brain:plasma ratio. At 15, 30 and $60 \mathrm{~min}$ after dauricine treatment, the dauricine brain:plasma ratio in the verapamil group was significantly wincreased compared with that of the control group $(P<0.01$, Fig. 7).

\section{Discussion}

The BBB, which is situated between the blood and the brain tissue, regulates the entry of drugs into the brain. The therapeutic effect or the toxicity of drugs to the CNS is dependent on the integrity of the BBB. The barrier function of the BBB originates from two major aspects: Firstly, the physiological structure, comprising brain microvessel endothelial cells, astrocytes and tight junctions between the endothelial cells, is the basis of the barrier function (12). Secondly, various types of transport proteins (13) at the BBB mediate the adsorption, excretion, distribution and elimination of drugs.

The results of this study demonstrated that at 15 min after dauricine administration, dauricine concentration in the brain reached a high level in the control and verapamil groups, which indicated that dauricine is able to cross the BBB. Furthermore, dauricine levels in the brain of the verapamil group were significantly higher than those in the control; however, verapamil did not affect dauricine levels in plasma, which suggested that the effect of verapamil on dauricine levels in the brain did not depend on the interference with the elimination of dauricine from the blood. In turn, it may be deduced that P-glycoprotein has an important role in the elimination of dauricine from the brain, as verapamil, an inhibitor of P-glycoprotein, was capable of increasing the levels of dauricine in the brain.

This study only evaluated the influence of P-glycoprotein on the levels of dauricine, and the effect of dauricine on P-glycoprotein expression was not taken into consideration. As a result, further studies are required to focus on whether dauricine is able to modulate the function or expression of P-glycoprotein.

In conclusion, the present study demonstrated that dauricine is able to pass the BBB. It was also shown that inhibiting P-glycoprotein was able to enhance dauricine levels in the brain. The results suggest that dauricine, originating from Traditional Chinese Medicine, may be a promising candidate for the treatment of CNS disorders.

\section{Acknowledgements}

The study was supported by the Natural Science Foundation of Heilongjiang Province of China (grant no. D201038).

\section{References}

1. Abbott NJ, Patabendige AA, Dolman DE, Yusof SR and Begley DJ: Structure and function of the blood-brain barrier. Neurobiol Dis 37: 13-25, 2010.

2. Bellavance MA, Blanchette M and Fortin D: Recent advances in blood-brain barrier disruption as a CNS delivery strategy. AAPS J 10: 166-177, 2008.

3. Mehdipour AR and Hamidi M: Brain drug targeting: a computational approach for overcoming blood-brain barrier. Drug Discov Today 14: 1030-1036, 2009.

4. He L and Liu GQ: Interaction of multidrug resistance reversal agents with P-glycoprotein ATPase activity on blood-brain barrier. Acta Pharmacol Sin 23: 423-429, 2002

5. Liu JY, Thom M, Catarino CB, Martinian L, Figarella-Branger D, Bartolomei F, Koepp M and Sisodiya SM: Neuropathology of the blood-brain barrier and pharmaco-resistance in human epilepsy. Brain 135: 3115-3133, 2012.

6. Pérez-Tomás R: Multidrug resistance: retrospect and prospects in anti-cancer drug treatment. Curr Med Chem 13: 1859-1876, 2006.

7. Qian JQ: Cardiovascular pharmacological effects of bisbenzylisoquinoline alkaloid derivatives. Acta Pharmacol Sin 23: 1086-1092, 2002.

8. Xia JS, Li Z, Dong JW, Tu H and Zeng FD: Dauricine-induced changes in monophasic action potentials and effective refractory period of rabbit left ventricle in situ. Acta Pharmacol Sin 23: 371-375, 2002

9. Tang XD, Zhou X and Zhou KY: Dauricine inhibits insulin-like growth factor-I-induced hypoxia inducible factor lalpha protein accumulation and vascular endothelial growth factor expression in human breast cancer cells. Acta Pharmacol Sin 30: 605-616, 2009.

10. Yang Z, Li C, Wang X, et al: Dauricine induces apoptosis, inhibits proliferation and invasion through inhibiting NF-kappaB signaling pathway in colon cancer cells. J Cell Physiol 225: 266-275, 2010.

11. Li YH and Gong PL: Neuroprotective effects of dauricine against apoptosis induced by transient focal cerebral ischaemia in rats via a mitochondrial pathway. Clin Exp Pharmacol Physiol 34: 177-184, 2007.

12. Begley DJ: Delivery of therapeutic agents to the central nervous system: the problems and the possibilities. Pharmacol Ther 104: 29-45, 2004.

13. Megard I, Garrigues A, Orlowski S, et al: A co-culture-based model of human blood-brain barrier: application to active transport of indinavir and in vivo-in vitro correlation. Brain Res 927: 153-167, 2002. 